\title{
Vulnerability, sensibility or coastal indicators? A preliminary analysis for a methodology of risk analysis
}

\author{
F. Taveira-Pinto \\ IHRH-FEUP, Department of Civil Engineering, University of Porto, \\ Portugal
}

\begin{abstract}
The strong coastal erosion, which has been increasing on many coasts, emerges as a consequence of different processes, acting in the land/sea interface area. These processes have their origin in either human or natural actions, all contributing to the degradation of coastal landscape. This deterioration is strongly connected to the intense human occupation of coastal areas, which transforms them into areas with a greater environmental vulnerability and risk, in relation to erosion and flood events.

The development of environmental vulnerability and risk methodologies aiming at the production of digital cartography, in order to support territorial planning in coastal areas, is also of great importance in the decision process.

The concept of vulnerability can also be understood as a sensibility analysis or coastal indicators. This paper intends to give not only a global perspective of the vulnerability/risk aspects, but also the new approaches running under the scope of some EU projects, particularly the EUROSION Project.
\end{abstract}

Keywords: vulnerability, coastal indicators, risk, uncertainty.

\section{Introduction}

The environmental vulnerability could be described as the response ability in a catastrophic situation. The bigger this response ability is, the lower the environmental vulnerability and damage will be.

The vulnerability of a coastal unit exposed to the sea actions (waves, tides, currents, flood, winds, etc) represents the sensibility to those actions expressed through hydromorphological changes. 
A natural shoreline will be more vulnerable and will recede more rapidly due to high tide and wave energy action, to a lack of natural "defence" in the case of lowlands and if there is high sediment transport deficit.

To define environmental risk is to characterise the potential adverse effects, resulting from the environmental dangers exposure and to the uncertainties related with the risk analysis process. When the damages can be measured, the risk is the occurrence probability of that action, multiplied by the perjury importance. Usually the undesirable consequences are not measurable. In that case the risk is equal to the probability itself.

The risk of a coastal unit is "the weighed average of the consequences of each action mode judged by the probability of each failure". The exposure risk of that unit to the sea actions depends not only on its vulnerability but also on the characteristics of the land use.

The natural risk is related with an extreme geophysical event that can cause certain damages, as a consequence of both the natural system and coastal zone interaction. An important factor that could affect the risk basis is the "random effect", which could, when facing an implicit danger, cause what is called the potential risk. If the risk is caused or increased by human activities it is called the induced risk.

\section{General evaluation}

The risk quantification process describes the use of some principles to obtain risk estimates, as a basis for the decision-making and risk management [3,6-8], within a responsive management framework, see Figure 1, [5].

The methodology to apply should include the following steps, see Figure 2, [4]:

- Potential risks identification and exposure evaluation;

- Vulnerability analysis;

- Risk quantification and uncertainty of its estimate.

The potential risks identification and exposure evaluation is related with the analysis of all existing elements from all factors detected in the study area, their distribution and evaluation, identifying those with a greater potential risk. The risk evaluation needs a clear understanding of all-important factors and their environmental influence on the local and surrounding physical systems. Special care should be taken on the more sensitive systems identification and on the short and long term exposure estimates. The vulnerability analysis aims at the identification, for each selected factor (figure 2), of the values that could be assumed for each case study. For that factor or physical process, a quantitative or qualitative ranking variable is obtained by this method.

This rating could be obtained or not, through models more or less sophisticated, namely for the hydrodynamic processes evaluation. The risk quantification for a specific risk analysis includes the results from the vulnerability analysis, the characteristics and possible failure modes on the coastal unit (beaches, dunes, cliffs, defence works) and on the land use near the 
shoreline, the alternative "scenarios" of urban expansion and the acceptable risk levels related with social and economic criteria [6].

The results from the vulnerability provide the occurrence probability of each factor value. The other elements provide for each occurrence the consequence of each failure mode, if they can be measured. From the obtained data through the vulnerability analysis, assuming the factor evaluation, the mean values must be used to assess the cyclic exposure related risks, as this measure is the best exposure estimate, during a certain period of time.

The maximum values are used for a short period risk analysis or for maximum potential risk estimates.

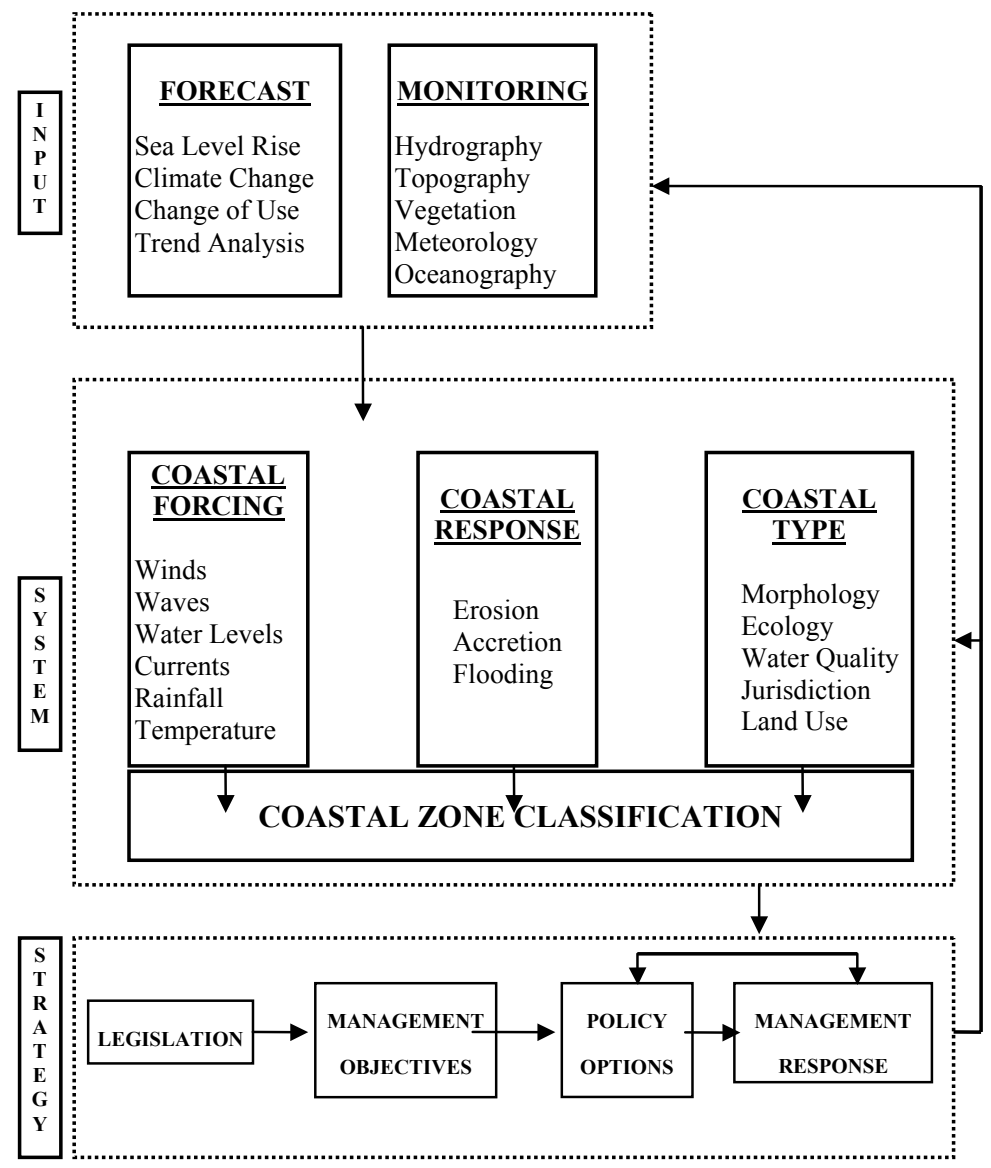

Figure 1: $\quad$ Responsive management framework (Townend [5]).

Risk analysis is the logical extension of sensitivity analysis when there is enough information to take probabilities and correlations into account explicitly and numerically. However analytical methods are sometimes impracticable and so simulation methods are practicable though time-consuming. The analysis needs more information than simple sensitivity tests. 


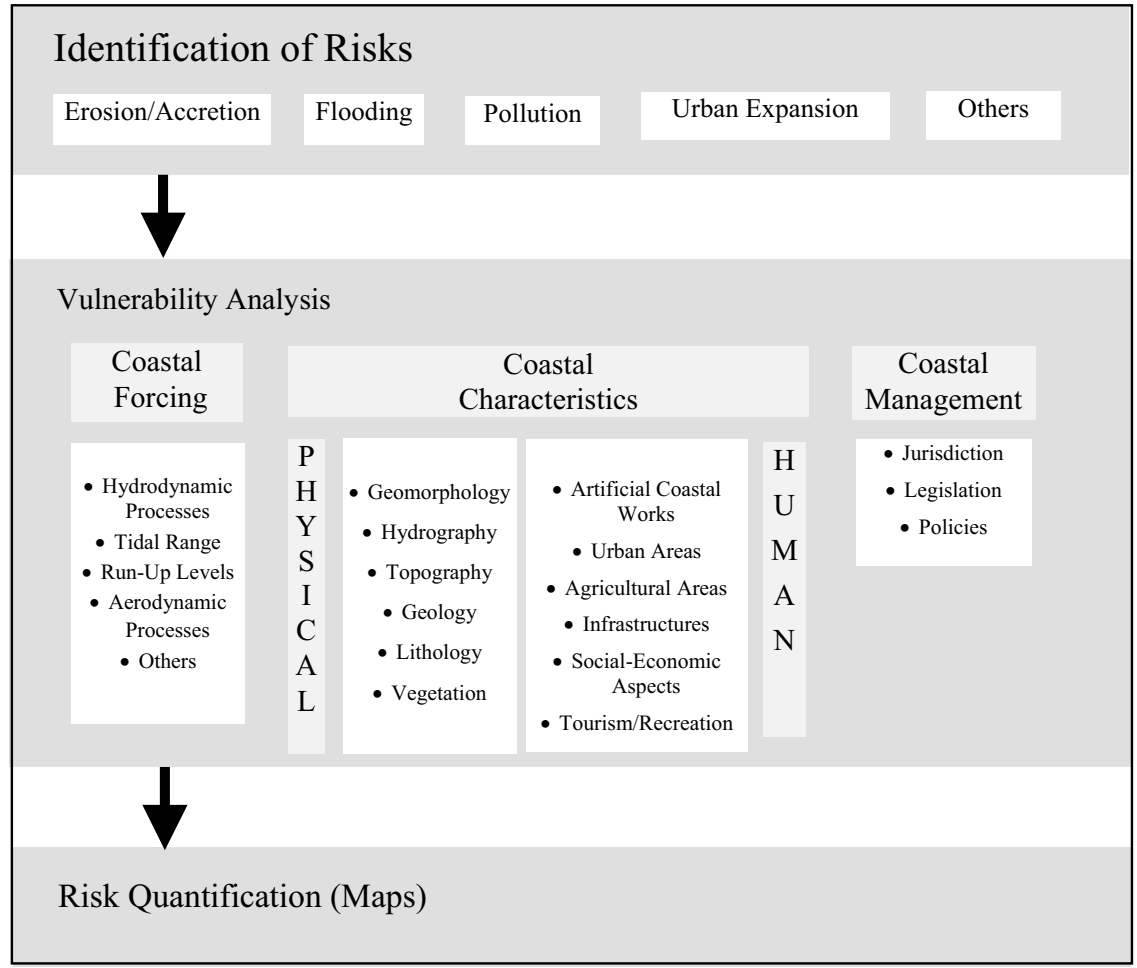

Figure 2: $\quad$ General methodology (Ferreira et al. [2]).

The first step is to identify the key independent parameters whose variations have significant effects on the outcome: this can, of course, be done by sensitivity tests. Each parameter used in the risk analysis should be a quantifiable one whose value is an arithmetic input into the calculation indicators.

Step 2 is to estimate each chosen parameter's probability distribution, from sophisticated statistical analysis or a reasonable estimate, remembering of course that high precision is impossible.

The third step, both a difficult and an important one, is to estimate correlations between the chosen variables.

If there is any functional reason or causal link that might tend to make one parameter deviate from its expected value under the same circumstances that cause another parameter to deviate, the strength of that tendency must be estimated or guessed in terms of the usual correlation coefficient. This applies either when one parameter is partially a cause of another or when they are both affected by a third phenomenon, a common cause, which is not one of the analysis parameters. The total number of independent and partially dependent variables should probably not exceed about ten.

Step 4 is to simulate the analysis (or run the model) a large number of times with different values of the chosen parameters each time. The values taken by 
each varying parameter, over the whole series, must conform to the probability distribution as defined in step two, and if correlations are in force the cross-correlations between parameters must also be as specified.

Finally, as Step 5, the results must be presented and interpreted. Through the probability distribution of the output, a parameter's values can be presented, as a histogram, as a cumulative curve or as a table of descriptive numbers such as mean, standard deviation, quartiles, deciles and extremes. Even when the varying input parameters are rectangular or have very skew distributions, the output parameter needs to have relatively smooth and symmetrical distributions, especially if correlation is weak. It is however, quite likely that the expected value of the indicator will be significantly different from the value that would be derived from a single calculation using the expected values of all input parameters especially if correlations are strong. Provided that the estimates are sensible this difference is not a delusion, and the risk analysis is a better decision guide than the single result even with sensitivity tests.

The last two steps (simulation and result presentation) can be made using the usual computer software such as the Monte-Carlo simulator method.

Risk analysis of this sort is a powerful tool. The experimentation should include changing the correlation coefficients, whose effect can be surprisingly strong. Though risk analysis is in other respects a step beyond sensitivity tests, it may be wise to do some sensitivity tests on the risk analysis coefficients. It is also useful to gain experience of the effects of having fewer or more parameters varying. If there are too many of them, and/or if the assumed correlations are too weak, their variations tend to cancel out in the computation and produce an unrealistically small spread in the output variable.

\section{Recent developments under the scope of the EU EUROSION project}

EUROSION [4] was a project commissioned by the General Directorate Environment of the European Commission, which will result in policy recommendations on how to manage coastal erosion in Europe in the most sustainable way.

One quarter of the European Union's coast is currently eroding despite the development of a wide range of measures to protect shorelines from eroding and flooding. The prospect of further sea level rises due to climate change and the heritage of mismanagement in the past - such as inappropriate infrastructure imply that coastal erosion will be a growing concern in the future.

EUROSION is based on the assumption that coastal erosion is a phenomenon that can never be completely controlled but can be managed in an economically and ecologically sustainable fashion.

The holistic view integrates:

- Natural and human-induced causes of erosion (storms, seismic movements, reduced sediment supply from rivers due to dams, coastal defences themselves, etc.); 
- Different uses of the coast - including both its terrestrial and marine parts - ranging from biodiversity and landscape conservation to tourism, industry and transport;

- Different levels of management - from local to European and regional seas;

- River basin considerations;

- Present concerns and long term perspectives (e.g. climate change).

A 4-step-process has been defined concerning the development of policy recommendations.

1 - Defining the scope and highlighting the trends of coastal erosion;

2 - Reviewing lessons learned from local shoreline management practices;

3 - Assessing the vulnerability of the European coastline;

4 - Integrating the various deliverables into the policy recommendations.

The Europe-wide database, defined under the scope of this project, does not provide any operational knowledge to support policy recommendations. Most of the data layers produced by the project need post-processing and combining to be converted into meaningful and action-oriented information, which will make sense for policy-makers.

Therefore, the approach proposed is twofold:

- A first step to establish a set of reference indicators, which provide a measurable "snapshot" of the major details of coastal erosion processes throughout Europe.

- A second step to set up - for each reference indicator - threshold values, which reflect the sensitivity of coasts to specific phenomena such as sea level rises, increased storms, or urbanization. The combined value of all indicators is expected to make it possible to zone coastal areas according to their overall vulnerability to coastal erosion.

The approach adopted to establish the set of reference indicators is based on the DPSIR model (Driving forces (D), Pressure (P), State (S), Impact (I), and Responses (R)), according to the European Environment Agency (EEA). Figure 3 briefly describes the two-step process - from data to information and from information to action - for assessing the vulnerability of European coasts to coastal erosion, and provides a tentative list of DPSIR indicators. This set of reference indicators should be maintained in the future as a tool for monitoring the effectiveness of policy implementation.

Based on the reference set of indicators, the EUROSION project has proposed a zoning of European coasts into vulnerable areas for which some specific actions should be recommended at different levels of administration and within a specific timeframe. More precisely, a geographical delineation of European coastal zones into the following four vulnerability classes is proposed:

- (A) Erosion Hotspots will characterize areas where economical, social and ecological assets at risk of eroding/flooding are maximal in spite of efforts to protect them. Related with eroding areas where:

$>$ Existing coastal defence and/or erosion management measures locally implemented do not counteract the coastline recession; 
Risk Analysis VI 283

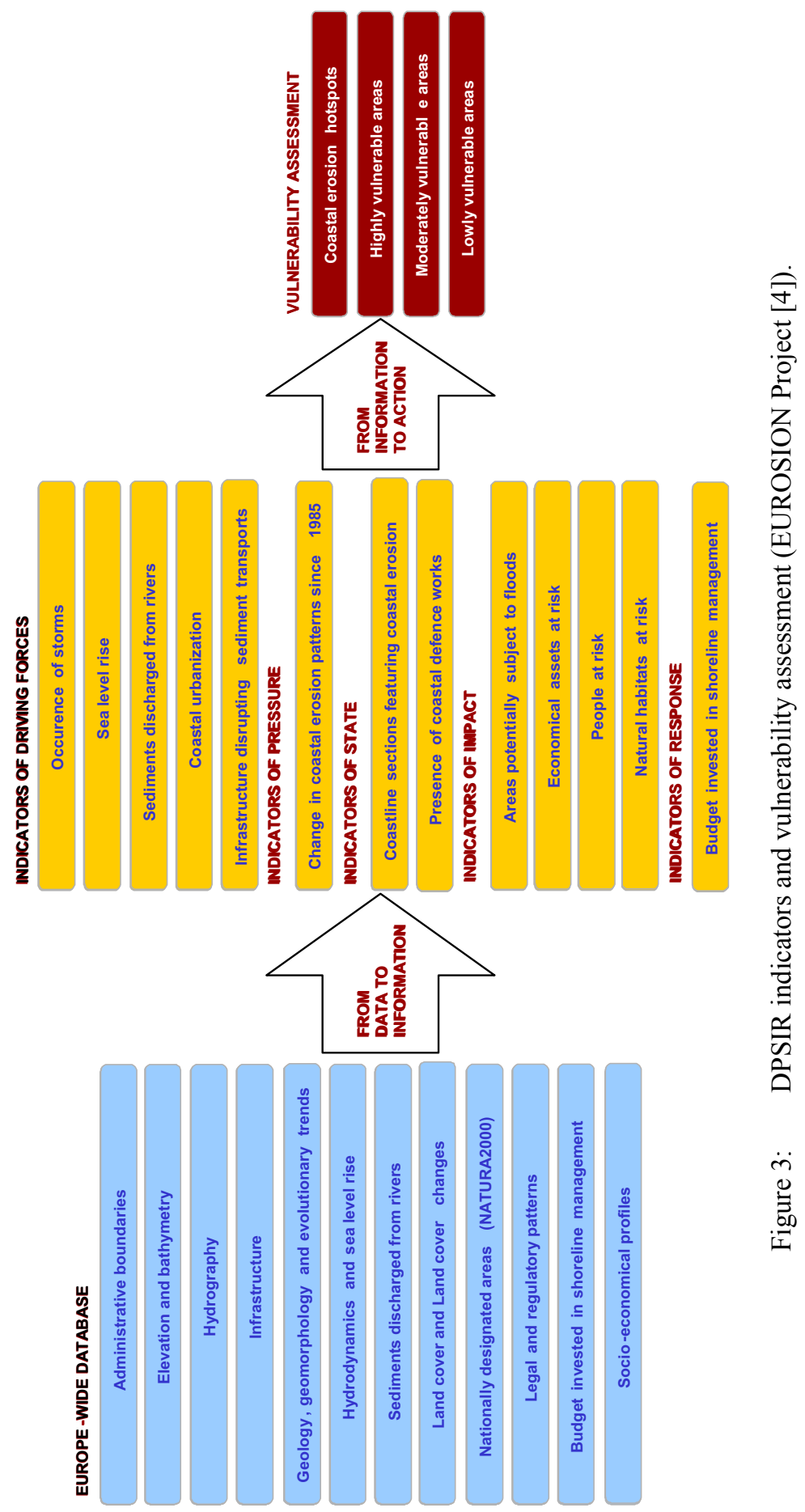

WIT Transactions on Information and Communication, Vol 39, (C) 2008 WIT Press www.witpress.com, ISSN 1743-3517 (on-line) 
Major economical, social and/or ecological assets are located less than $1 \mathrm{~km}$ from the shoreline.

- (B) Highly Vulnerable Areas will characterize eroding areas with important economical, ecological and social assets which have not benefited so far from any specific erosion management measure, but on which current trends are likely to aggravate the impact of coastal erosion. Related with eroding areas where:

$>$ No existing coastal defence and/or erosion management measures are implemented locally to counteract the coastline recession;

> Major economical, social and/or ecological assets are located less than $1 \mathrm{~km}$ from the shoreline;

$>$ Current trends - such as sea level rises or urbanization - are likely to aggravate coastal erosion processes.

- (C) Moderately Vulnerable Areas will characterize eroding or non-eroding areas with important assets that do not seem - at least, at first sight - to be immediately threatened by coastal erosion processes. Related with eroding areas where:

$>$ No existing coastal defence and/or erosion management measures are implemented locally to counteract the coastline recession;

$>$ Major economical, social and/or ecological assets are located less than $1 \mathrm{~km}$ from the shoreline;

$>$ Current trends - such as sea level rises or urbanization - are not likely to aggravate coastal erosion processes.

Or related with non-eroding areas where:

$>$ Coastal erosion processes are locally counteracted by coastal defence and/or erosion management measures;

$>$ Major economical, social and/or ecological assets are located less than $1 \mathrm{~km}$ from the shoreline.

- (D) Lowly Vulnerable Areas will characterize areas with - at first sight - no major economical, social and ecological assets. No particular action is necessary but national and local institutions are encouraged to support research and monitoring activities. Eroding or non-eroding areas where no major economical, social and/or ecological assets are located less than $1 \mathrm{~km}$ from the shoreline.

For the first three levels, should no adequate coastal defence and/or erosion management policy already be designed for such areas, further investigations should be undertaken in order to provide more detailed information on the shoreline evolution and assets at risk in these particular areas, as well as to determine which policy option is the most appropriate for these areas for the coming years.

To avoid misunderstandings about this vulnerability assessment, it is important to bear in mind the following three limitations of such an approach:

- The vulnerability assessment is mainly based on a GIS processing of the Europe-wide database and a comparison with the Corine Coastal Erosion 
database; as the latter represents the situation for the period 1985-1990, the time span of the trends is ca. 10-15 years. As such, it depends on the quality of data within the database, which is not always guaranteed. It has to be seen as an attempt to provide the best overview of European coasts vulnerability to coastal erosion given the level of knowledge available in 2002 at a European-scale (1:100.000). Therefore, some errors are impossible to anticipate and could not be counteracted in the timeframe of the project.

- The concept of "major" economical, social, and ecological assets on the coastal zone is subjective. This does not preclude that some assets of sub-national importance may require immediate erosion management measures.

The vulnerability assessment cannot be prescriptive in terms of policy options, as the level of details featured by the Europe-wide database is precluding any acceptable recommendations in that sense. The vulnerability assessment has to be seen as an instrument to set priorities in terms of time and investments and suggest the most appropriate level of administration (European, national, local) to manage some areas with the attention they deserve. In any case, further investigations are needed to confirm and better quantify the risks, helping decision-makers in their policy decision role.

The term 'Vulnerability Assessment' was not always understood. In regard to this, a "Sensitivity Assessment" or "Coastal Typology in relation with Erosion" was developed, according to the following coastal classification:

(D) Hard rock elevated coast, with no flooding, no overall erosion, dynamic coast eroding, semi-stable/accreting, no assets at risk, good sediment supply;

(C) Less resistant rock, dynamic coast semi-stabled and eroding, some assets at risk - property/life, moderate sediment supply;

(B) Unstable cliffs, low-lying areas mostly below mean sea level, dynamic coast with erosion, and assets at risk;

(A) Highly unstable cliffs, low-lying areas all below mean sea level, high value of assets at risk, sediment deficit

Regarding the application of the DPSIR model, the adoption of more simplified model is suggested: Pressure, State and Response.

The integration of this approach, once the 'Classification of the Coast' is defined, might allow one to know that a coastal area is under certain pressures, whether it has a certain state and the different policy options on how to respond and act. In this sense, an overview of the options can be given with a description on what needs to be done. As an example for a certain state of the coast and an application of a certain option it is advisable to further develop risk assessments, environment impact assessment (EIA), strategic environment assessment (SEA) and economical assessments.

\section{Uncertainty and risk}

Vulnerability and risk concern future actions and all cumulative statements about such actions and their consequences have a significant degree of uncertainty. Whenever there is more than one possible outcome of an action or investigation, 
there is uncertainty. Usually there is some information about the relative probability of each outcome, even if it is only subjective or rough. So, the words risk and uncertainty are sometimes used in special senses.

Uncertainty is an unknown probability, undefined, or with no consensus. It can be statistical (repeatable) or non-statistical (subjective). Risk is the known or estimated probability distribution.

The important concept is that there are two sorts of statement about any outcome or parameter: a simple statement of its magnitude and a more complex statement about the probabilities of different outcomes. The latter statement contains much more information than the former (information can be expensive), and it is also much more useful. Probability information converts unquantified uncertainty into quantified uncertainty (sometimes called risk):

Unquantified uncertainty + Probability information $=$ Quantified uncertainty

Probability estimates can be entirely subjective; other probability estimates can be, in varying degrees, objective, to indicate fairly precisely the degree of accuracy or uncertainty of the estimate. Relative precision and uncertainty are quantified by statistical probability distributions, which are described by histograms, central measures, and dispersion measures.

\section{Final remarks}

Is the DPSIR process and vulnerability assessment compliable with the perspective of national viewpoints? Is this approach sufficient or should it be complemented by a more holistic approach of sediment budgets and opportunities for nature on a larger scale (taking into account adjacent coastal sectors)?

The treatment of uncertainty is a complex subject in which ideas are developing and changing, so that there is much to be gained by trying relatively new techniques. At the very least every simulation should have a few sensitivity tests, thoughtfully designed and carefully and transparently reported. The base case should incorporate the best estimates of all parameters, and any deliberate caution or pessimism in estimating should be identified and reported separately. Deviations from the base case should preferably be related to meaningful physical situations or thresholds rather than to arbitrary percentages.

For major analyses or projects with potential environmental irreversible consequences, consideration should be given to carrying out a formal risk analysis using simulation methods. The analyst should, however, first gain experience with the use of this tool, and draw on the experience of someone who has used it before.

All analysis and discussion of uncertainty demonstrates the value of information and the potential for feedback from analysis to design. An analysis of uncertainty carried out early in a project's development even with crude estimates, may have great value in guiding the design or re-design of the project, perhaps incorporating conscious risk management features or a focused effort to achieve flexibility and robustness in critical areas. 


\section{Acknowledgement}

The author wishes to acknowledge EUCC-The Coastal Union, for permission to use data from reference [4], to which the author has contributed.

\section{References}

[1] C. Coelho, R. Silva, F. Veloso-Gomes, Taveira Pinto, F., 2006, A Vulnerability Analysis Approach for the Portuguese West Coast, Risk Analysis 2006, Fifth International Conference on Computer Simulation in Risk Analysis and Hazard Mitigation, V. Popov and C. A. Brebbia (editors), Wessex Institute of Technology, Malta, June, pp.251-262, ISSN 1743-3541;

[2] Ferreira, J.C.; Alves, F.; Taveira Pinto, F., 1997, Vulnerability and Risk in the Portuguese Coastal Zone-A Methodology of Analysis, $6^{\text {th }}$ EUCC International Conference Coastlines'97, European Union for Coastal Conservation, June, Naples, Italy, pp.64 (Edition in CD);

[3] F. Veloso Gomes, F. Taveira Pinto, 1994, Urban Expansion in High Risk Northwest Coastal Areas of Portugal, September, International Symposium "Littoral' 94", Lisbon; "Littoral' 94" Proceedings, Eurocoast - Portugal Association Edition, G. Soares de Carvalho and F. Veloso Gomes Editors, pp. 981-996;

[4] Living with coastal erosion in Europe. Major findings and Policy Recommendations. Service contract B4-3301/2001/329175/MAR/B3, "Coastal erosion - Evaluation of the need for action", Directorate General Environment, European Commission, March 2004.

[5] Townend, I.H., 1993, Coastal Management. Coastal, Estuarial and Harbour Engineer's Reference Book.

[6] Veloso Gomes, F., Taveira Pinto, F., M. Papadatos, Vulnerability and Risk Assessment of Urban Areas Exposed to Coastal Forcing. Oral Presentation at Littoral'96. Portsmouth. Eurocoast-UK.

[7] Veloso Gomes, F., Taveira Pinto, F., 1997, Portuguese Urban Waterfronts Expansion near Coastal Areas, "Environmental Challenges in an Expanding Urban World and the Role of Emerging Information Technologies" Congress, Edit. João Reis Machado and Jack Ahern, Junho, Lisboa, pp.189 198, ISBN-972-97406-0-7;

[8] Veloso Gomes, F.; Taveira Pinto, F.; das Neves, L.; Barbosa, J.P., Coelho, C., 2002, High Risk in the in the NW Portuguese Coast: Douro River-Cape Mondego, Proceedings of the Sixth International Conference LITTORAL 2002 (ed. F. Veloso Gomes, F. Taveira Pinto \& Luciana das Neves), Vol. II, pp. 411-421. ISBN 972-8558-09-0; 